\title{
The Impact of Socioeconomic Status on the Clinical Outcomes of COVID-19; a Retrospective Cohort Study
}

\author{
Christine Little ${ }^{1} \cdot$ Mathilda Alsen $^{2} \cdot$ Joshua Barlow $^{1} \cdot$ Leonard Naymagon $^{3} \cdot$ Douglas Tremblay $^{3} \cdot$ Eric Genden $^{2}$. \\ Samuel Trosman ${ }^{2} \cdot$ Laura lavicoli $^{4} \cdot$ Maaike van Gerwen $^{2,5}$ (I)
}

Accepted: 29 October 2020 / Published online: 2 January 2021

(c) Springer Science+Business Media, LLC, part of Springer Nature 2021

\begin{abstract}
There have been limited data assessing the influence of disadvantaged socioeconomic status (SES) on the incidence and clinical outcomes of COVID-19 patients within the diverse communities of the United States. Here, we aim to investigate the association between poverty level, as an indicator of SES, and COVID-19 related clinical outcomes including hospitalization and all-cause mortality. This retrospective cohort study included 3528 patients with laboratory confirmed COVID-19 seen at a large New York City health system between March 1, 2020 and April 1, 2020. Data for neighborhood level poverty was acquired from the American Community Survey 2014-2018 and defined as the percent of residents in each ZIP code whose household income was below the federal poverty threshold (FPT): $0 \%$ to $<20 \%$ below FPT (low poverty) and $>20 \%$ below FPT (high poverty). COVID-19 positive patients who resided in high poverty areas were significantly younger, had a higher prevalence of comorbidities and were more likely to be of female gender or a racial minority when compared to individuals living in low poverty areas. Residence in a high poverty area was not associated with an increased risk of COVID-19 related hospitalization and was found to be associated with a decreased risk of in-hospital mortality. This study suggests the existence of an unequal socioeconomic gradient in the demographic and clinical presentation of COVID-19 patients including differences in age, gender and race between poverty groups. Further studies are needed to fully assess the intersectionality of SES with the COVID-19 pandemic.
\end{abstract}

Keywords COVID- $19 \cdot$ Poverty $\cdot$ SARS-CoV-2 $\cdot$ Socioeconomic status

Electronic supplementary material The online version of this article (https://doi.org/10.1007/s10900-020-00944-3) contains supplementary material, which is available to authorized users.

Maaike van Gerwen

maaike.vangerwen@mountsinai.org

1 Department of Medical Education, Icahn School of Medicine at Mount Sinai, New York, USA

2 Department of Otolaryngology-Head and Neck Surgery, Icahn School of Medicine at Mount Sinai, One Gustave L. Levy Place, New York, NY 10029, USA

3 Division of Hematology and Medical Oncology, Icahn School of Medicine at Mount Sinai, New York, USA

4 Department of Emergency Medicine, Icahn School of Medicine at Mount Sinai, New York, USA

5 Institute for Translational Epidemiology, Icahn School of Medicine at Mount Sinai, New York, USA

\section{Introduction}

The outbreak of severe acute respiratory syndrome coronavirus 2 (SARS-CoV-2) has rapidly evolved into a global pandemic since it first emerged in late 2019. Continued monitoring of the incidence and clinical outcomes of patients diagnosed with Coronavirus Disease 2019 (COVID-19) is vital to understand the epidemiological burden of SARS$\mathrm{CoV}-2$ within the United States. Several risk factors have been associated with COVID-19 severity including old age, male gender and patients with underlying chronic conditions or comorbidities [1]. Specifically, data from the clinical literature has indicated that persons with obesity, diabetes, hypertension or coronary artery disease experience poorer clinical outcomes including need for invasive mechanical ventilation and death [2-4].

Recent evidence has suggested that racial and ethnic minorities are disproportionately affected by COVID19. Black and Hispanic individuals are more likely to test 
positive for SARS-CoV-2 and are at an increased risk of hospital admission for COVID-19 compared to white individuals [5-7]. It is not yet understood whether such disparities are driven by differences in underlying health conditions, geographic location or social deprivation. The United States Centers for Disease Control and Prevention (CDC) has suggested that racial disparities in COVID-19 outcomes may be due, in part, to socioeconomic disadvantages that place low-income individuals at a higher risk of infection [8]. Individuals with a low income are more likely to work "essential jobs" that require continued work outside the home, to live in multi-generational households or crowded housing conditions and to lack jobs that offer paid sick leave [8].

Disadvantaged socioeconomic status (SES) has previously been associated with an increased risk of hospital admissions for respiratory infections [9-11]. A recent study from the Chennai megacity of India found that low-SES areas experienced a higher incidence of COVID-19 infections, suggesting that COVID-19 may disproportionately affect low-income individuals, although they did not examine SES as a risk factor for COVID-19 morbidity and mortality [12]. Despite these indications that disadvantaged SES may be an important risk factor for COVID-19 infection, there remains little research investigating the relationship between COVID-19 and SES within the diverse communities of the United States, especially in regards to SES as a risk factor for COVID-19 morbidity and mortality. Thus, the influence of socioeconomic factors on the incidence and clinical outcomes of COVID-19 has been deemed a research priority, in order to further elucidate the epidemiological burden of SARS-CoV-2 within the United States and inform public health prevention measures [13]. Here, we aim to investigate the association between poverty level, as indicator of SES, and COVID-19 related clinical outcomes, including hospitalization and all-cause mortality.

\section{Methods}

\section{Study Population}

This retrospective cohort study included all patients seen at a large New York City health system $(n=4343)$ who tested positive for COVID-19 on a reverse-transcriptase-polymerase-chain-reaction (RT-PCR) SARS-CoV-2 assay of a nasopharyngeal swab specimen between March 1, 2020 and April 1, 2020. Both hospitalized and ambulatory patients were included. Patients were excluded if they were less than 18 years old $(n=55)$ or had insufficient clinical documentation available for review, including confidential patient records $(n=585)$. Additionally, patients for whom ZIP code information was not available or those who did not reside within the New York City (NYC) residential ZIP code area were excluded from the study $(\mathrm{n}=175)$, resulting in a final study population of 3528 COVID-19 positive patients. This study was reviewed and approved by the Institutional Review Board of the Icahn School of Medicine at Mount Sinai.

\section{Data Collection}

The medical records of all patients were retrospectively reviewed and relevant clinical data extracted and securely stored using Research Electronic Data Capture software (REDCap, Vanderbilt University). The extracted data included the following: demographic characteristics (age, sex, patient-reported race, ZIP code), medical comorbidities, patient-reported smoking status and body mass index (BMI) with cut-offs used for normal weight $\left(<25 \mathrm{~kg} / \mathrm{m}^{2}\right)$, overweight $\left(25-30 \mathrm{~kg} / \mathrm{m}^{2}\right)$ and obese $\left(>30 \mathrm{~kg} / \mathrm{m}^{2}\right)$. Patientreported race was categorized in the following groups: non-Hispanic white, non-Hispanic black or "other race" for patients who identified as Asian, Hispanic, American Indian or Alaska Native, Native Hawaiian or Pacific Islander; patients for whom race information was not available were categorized as "unknown." Survival time was calculated as the days between a positive result on a RT-PCR SARS$\mathrm{CoV}-2$ assay of a nasopharyngeal swab specimen and last patient follow-up or date of death. The primary outcomes of interest included hospital admission and all-cause mortality. Data on the primary outcomes of interest was collected up to May 13th, 2020, which provided a minimum follow-up time of six weeks for each patient.

\section{Poverty Levels}

Data for neighborhood-level poverty, the primary predictor, was acquired from the American Community Survey (ACS) 2014-2018 for 214 New York City (NYC) residential ZIP codes and assigned to patients' reported ZIP code at date of COVID-19 diagnosis. Neighborhood-level poverty was chosen as our primary indicator of SES, which is in accordance with recommendations from the NYC Health Department (NYC DOHMH), who have validated the use of ZIP code level census data as a measure of area-based SES [14]. Following NYC DOHMH guidelines, neighborhood-level poverty was defined as the percent of residents in each ZIP code whose household income was below the federal poverty threshold (FPT) [14]. The low poverty group consisted of areas in which $0 \%$ to $<20 \%$ of residents lived below the FPT and the high poverty group consisted of areas in which $>20 \%$ residents lived below the FPT. ZIP code level income indicators, such as percent living below the FTP, have been independently validated across several studies as an appropriate method of calculating area-based SES [15-17]. Furthermore, ZIP code level income indicators have been shown to be as effective as other commonly used 
measures of SES, such as highest self-reported education level, in assessing the impact of SES disparities on clinical outcomes [16].

\section{Statistical Analysis}

Demographic and clinical characteristics of COVID-19 positive patients were compared between low poverty and high poverty groups using a two-sided t-test for age and chisquared tests for the categorical variables. Within the hospitalized group, demographic and clinical characteristics were compared between low and high poverty groups in the same manner. Categorical measures are presented as percentages and continuous measures are presented as means and standard deviations.

The association between poverty and hospitalization was assessed using unadjusted and adjusted logistic regression models. Variables found to be significantly different between low and high poverty in the univariable analysis were adjusted for in multivariable logistic regression models $(\mathrm{p}<0.05)$. Results are presented as odds ratio (OR) with $95 \%$ confidence intervals ( $95 \% \mathrm{CI}$ ). Within the group of hospitalized patients, we performed time-to-event analysis for all-cause mortality. Censoring was applied for patients with a survival time exceeding 50 days to avoid a small number of patients at risk for the event of interest. Results were reported as a Kaplan-Meier curve with significance indicated by using log-rank test. A Cox proportional hazard model of all-cause mortality with robust sandwich variance estimates of standard errors was performed, adjusted for variables found to be significantly different between the low and high poverty groups of hospitalized patients in the univariable analysis $(\mathrm{p}<0.05)$. Results were reported as hazard ratio (HR) with $95 \%$ CI. We additionally assessed gender-specific variations in mortality through stratification of the model between male and female patient groups. All statistical analyses were performed using SAS University Edition (SAS Institute Inc., Cary, NC).

\section{Results}

\section{Characteristics of COVID-19 Positive Patients}

The mean age of the 3528 COVID-19 positive patients included in our final study population was $57.0( \pm 18.2)$ years. The study population was predominantly male (57.0\%); $25.7 \%$ was non-Hispanic white, $27.6 \%$ non-Hispanic black and $41.0 \%$ of other race. (Table 1) The mean age of COVID-19 positive patients in the high poverty group was significantly younger compared to the low poverty group ( 55.2 vs. 58.3 years, $\mathrm{p}<0.001)$; nearly a quarter of patients in the high poverty group were under 40 years of age, compared to only $19.7 \%$ of those in the low poverty group $(\mathrm{p}<0.001)$. Individuals in the high poverty group were significantly more likely to be obese (defined as a BMI > 30) and had a significantly higher prevalence of congestive heart failure (CHF), peripheral vascular disease (PVD), asthma, prior venous thromboembolism (VTE), diabetes and chronic kidney disease (CKD) than patients in low poverty areas. The percentage of non-Hispanic black patients living in high poverty areas was twice that of nonHispanic white patients. There was no significant difference in rates of hospital admission for COVID-19 between low and high poverty groups $(\mathrm{p}=0.699)$.

\section{Characteristics and Outcomes of Hospitalized Patients}

Of the 3528 included patients who tested positive for COVID-19, 1904 (54\%) required admission to the hospital. (Table 1) The mean age of admitted patients in the high poverty group was significantly younger compared to the low poverty group ( 66.8 vs. 62.3 years, $\mathrm{p}<0.001)$. (Table 2 ) $41.2 \%$ of patients in the high poverty group were aged 60 years or younger, compared to only $31.8 \%$ of patients in the low poverty group $(\mathrm{p}<0.001)$; patients in the high poverty group were nearly twice as likely to be under the age of 40 as those in the low poverty group. Patients in the high poverty group were significantly more likely to be obese than those in the low poverty group $(p=0.043)$. Individuals in the high poverty group had a significant higher prevalence of asthma, prior VTE and CKD than those in low poverty. Additional comorbidities reported in Table 1 were assessed among hospitalized patients but none were found to differ significantly between poverty groups and are therefore not included in Table 2.

Of patients hospitalized for COVID-19, 497 (26.1\%) required invasive mechanical ventilation and 595 (31.3\%) died. A significantly greater percentage of hospitalized patients in the low poverty group than the high poverty group required invasive mechanical ventilation (29\% vs. $21.7 \%, \mathrm{p}<0.001)$. The unadjusted case-fatality rate for patients in the low poverty group was $36.7 \%$ compared with $23 \%$ of patients among the high poverty group $(\mathrm{p}<0.001)$. The mean time to death was similar across poverty groups among deceased patients. (Table 2)

\section{Hospitalization}

Residence in a high poverty area was not independently associated with an increased odds of admission $\left(\mathrm{OR}_{\mathrm{adj}}\right.$, $0.98 ; 95 \%$ confidence interval [CI], 0.83-1.17). (Table 3 ) However, adjusted analysis revealed that non-Hispanic black patient were more likely to be hospitalized compared to non-Hispanic white patients $\left(\mathrm{OR}_{\mathrm{adj}}, 1.34 ; 95 \%\right.$ 
Table 1 Demographic and clinical characteristics of COVID-19 positive patients

\begin{tabular}{|c|c|c|c|c|}
\hline Characteristic & All $(n=3528)$ & Low poverty $(n=2090)$ & High poverty $(n=1438)$ & $P$ value \\
\hline Age (years \pm SD) & $57.03( \pm 18.2)$ & $58.3( \pm 18.2)$ & $55.2( \pm 18.1)$ & $<0.001$ \\
\hline Age groups (years) & & & & $<0.001$ \\
\hline$<40$ & $762(21.6)$ & $411(19.7)$ & $351(24.4)$ & \\
\hline $40-60$ & $1088(30.8)$ & $674(32.2)$ & $486(33.8)$ & \\
\hline$>60$ & $1678(47.5)$ & $1005(48.1)$ & $601(41.8)$ & \\
\hline Gender & & & & $<0.001$ \\
\hline Male & $1930(54.7)$ & $1197(57.3)$ & $733(51.0)$ & \\
\hline Female & $1598(45.3)$ & $893(42.7)$ & 705 (49.0) & \\
\hline Race & & & & $<0.001$ \\
\hline Non-Hispanic white & 908 (25.7) & $677(32.4)$ & $231(16.1)$ & \\
\hline Non-Hispanic black & 974 (27.6) & $516(24.7)$ & 458 (31.9) & \\
\hline Other & $1445(41.0)$ & $760(36.4)$ & $685(47.6)$ & \\
\hline Unknown & $201(5.7)$ & $137(6.6)$ & $64(4.5)$ & \\
\hline Smoking status & & & & 0.166 \\
\hline Current & $185(5.2)$ & $101(4.8)$ & $84(5.8)$ & \\
\hline Former & $696(19.7)$ & $404(19.3)$ & $292(20.3)$ & \\
\hline Never & $2039(57.7)$ & $1238(59.2)$ & $801(55.7)$ & \\
\hline Unknown & $608(17.2)$ & $347(16.6)$ & $261(18.2)$ & \\
\hline $\mathrm{BMI}>30^{*}$ & $1020(28.8)$ & $548(26.2)$ & $472(32.8)$ & $<0.001$ \\
\hline \multicolumn{5}{|l|}{ Comorbidities } \\
\hline \multicolumn{5}{|l|}{ Cardiovascular disease } \\
\hline Hypertension & $1589(45)$ & 931 (44.6) & $658(45.8)$ & 0.477 \\
\hline Atrial fibrillation & $294(8.3)$ & $173(8.3)$ & $121(8.4)$ & 0.885 \\
\hline Coronary artery disease & $426(12.1)$ & $260(12.4)$ & $166(11.5)$ & 0.422 \\
\hline Congestive heart failure & $280(7.9)$ & $149(7.1)$ & $131(9.1)$ & $\mathbf{0 . 0 3 3}$ \\
\hline PVD & $135(3.8)$ & $68(3.3)$ & $67(4.7)$ & $\mathbf{0 . 0 3 3}$ \\
\hline \multicolumn{5}{|l|}{ Chronic respiratory disease } \\
\hline Asthma & 407 (11.5) & $198(9.5)$ & $209(14.5)$ & $<0.001$ \\
\hline COPD & $161(4.6)$ & $95(4.6)$ & $66(4.6)$ & 0.951 \\
\hline \multicolumn{5}{|l|}{ Metabolic disease } \\
\hline Diabetes & $1012(28.7)$ & $563(26.9)$ & $449(31.2)$ & 0.006 \\
\hline Hypothyroidism & $238(6.8)$ & $153(7.3)$ & $85(5.9)$ & 0.101 \\
\hline CVA/TIA & $208(5.9)$ & $124(5.9)$ & $84(5.8)$ & 0.910 \\
\hline Prior VTE & $123(3.5)$ & $56(2.7)$ & $67(4.7)$ & 0.002 \\
\hline Chronic kidney disease & $336(9.5)$ & $166(7.9)$ & $170(11.8)$ & $<0.001$ \\
\hline Cancer & $295(8.4)$ & $182(8.7)$ & $113(7.9)$ & 0.370 \\
\hline Cirrhosis & $55(1.6)$ & $31(1.5)$ & $24(1.7)$ & 0.662 \\
\hline Dementia & $200(5.6)$ & $126(6.0)$ & $74(5.2)$ & 0.265 \\
\hline Admitted to Hospital & $1904(54.0)$ & $1140(54.6)$ & $764(53.1)$ & 0.699 \\
\hline
\end{tabular}

Bold values indicate statistical significance $(\mathrm{p}<0.05)$

$B M I$ body mass index, $C O P D$ chronic obstructive pulmonary disease, CVA/TIA cerebral vascular accident or transient ischemic attack, $P V D$ peripheral vascular disease, $S D$ standard deviation, VTE: venous thromboembolism

*683 patients had unknown or missing BMI data
CI, 1.07-1.69). The odds of hospitalization were even greater among non-white patients of "other" race when compared to non-Hispanic white patients $\left(\mathrm{OR}_{\mathrm{adj}}, 1.79\right.$; $95 \%$ CI, $1.45-2.20)$.

\section{All-Cause Mortality}

Survival was significantly better for patients in the high poverty group vs. those in the low poverty group 
Table 2 Clinical characteristics and outcomes of patients hospitalized for COVID-19

\begin{tabular}{|c|c|c|c|c|}
\hline Characteristic or clinical outcome & All $(n=1904)$ & Low poverty $(n=1140)$ & High poverty $(n=764)$ & $P$ value \\
\hline Age (years $\pm S D)$ & $65.8( \pm 16.0)$ & $66.8( \pm 15.7)$ & $62.3( \pm 16.8)$ & $<0.001$ \\
\hline Age groups (years) & & & & $<0.001$ \\
\hline$<40$ & $151(7.9)$ & $68(6.0)$ & $83(10.9)$ & \\
\hline $40-60$ & $532(27.9)$ & $294(25.8)$ & $238(31.1)$ & \\
\hline$>60$ & $1221(64.1)$ & $778(68.2)$ & $443(58.0)$ & \\
\hline Gender & & & & 0.115 \\
\hline Male & $1118(58.7)$ & $686(60.2)$ & $432(56.5)$ & \\
\hline Female & $786(41.3)$ & $454(39.8)$ & $332(43.5)$ & \\
\hline Race & & & & $<0.001$ \\
\hline Non-Hispanic white & $459(24.1)$ & $345(30.3)$ & $114(14.9)$ & \\
\hline Non-Hispanic black & $523(27.5)$ & $268(23.5)$ & $255(33.4)$ & \\
\hline Other & 835 (43.9) & $473(41.5)$ & $362(47.4)$ & \\
\hline Unknown & $87(4.6)$ & $54(4.7)$ & $33(4.3)$ & \\
\hline $\mathrm{BMI}>30 *$ & $653(36.6)$ & $367(34.7)$ & $286(39.3)$ & 0.043 \\
\hline \multicolumn{5}{|l|}{ Comorbidities $^{\dagger}$} \\
\hline Asthma & $218(11.5)$ & $113(9.9)$ & $105(13.7)$ & 0.010 \\
\hline Prior VTE & $83(4.4)$ & $38(3.3)$ & $45(5.9)$ & 0.007 \\
\hline Chronic kidney disease & $285(15)$ & $146(12.8)$ & $139(18.2)$ & 0.001 \\
\hline \multicolumn{5}{|l|}{ Hospital Course } \\
\hline \multicolumn{5}{|l|}{ Clinical measures } \\
\hline $\begin{array}{l}\text { Mechanical ventilation } \\
\text { required }\end{array}$ & $497(26.1)$ & $331(29.0)$ & $166(21.7)$ & $<0.001$ \\
\hline Bleeding event & $30(1.6)$ & $15(1.3)$ & $15(2.0)$ & 0.266 \\
\hline Overt thrombosis & $47(2.5)$ & $30(2.6)$ & $17(2.2)$ & 0.575 \\
\hline Renal replacement therapy & $128(6.7)$ & $86(7.5)$ & $42(5.5)$ & 0.081 \\
\hline \multicolumn{5}{|l|}{ Clinical outcomes } \\
\hline Died & $595(31.3)$ & $419(36.7)$ & $176(23.0)$ & $<0.001$ \\
\hline Alive at last follow up & $1309(68.7)$ & $721(63.3)$ & $588(77.0)$ & $<0.001$ \\
\hline $\begin{array}{l}\text { Mean time to death (days } \pm \\
\text { SD) }\end{array}$ & $10.2( \pm 8.7)$ & $9.8( \pm 8.6)$ & $11.2( \pm 8.8)$ & 0.066 \\
\hline $\begin{array}{l}\text { Mean length of follow up for } \\
\text { surviving patients (days } \pm \text { SD) }\end{array}$ & $21.38( \pm 13.50)$ & $20.87( \pm 13.5)$ & $22.01( \pm 13.4)$ & 0.125 \\
\hline
\end{tabular}

Bold values indicate statistical significance $(\mathrm{p}<0.05)$

$B M I$ body mass index, $S D$ standard deviation, $V T E$ venous thromboembolism

*118 patients had unknown or missing BMI data

Only comorbidities that were found to differ significantly between poverty groups in patients hospitalized with COVID-19 are reported. All other comorbidities reported in Table 1 were assessed and none found to be significant

¿Calculation of mean time to death included only patients who were deceased at the time of last follow up

$(\mathrm{p}<0.001)$. (Fig. 1) Residence in a high poverty area was associated with a decreased risk of mortality compared to residence in a low poverty area $\left(\mathrm{HR}_{\mathrm{adj}}, 0.61 ; 95 \% \mathrm{CI}\right.$ $0.51-0.73$ ). (Table 3) Stratification by gender showed that the decreased risk of mortality among individuals in the high poverty group existed for male patients (HR, $0.5 ; 95 \%$ CI, 0.039-0.64) but not for female patients ((HR, 0.83; 95\% CI, 0.62-1.11). (Supplementary Table 1) No difference was found in risk of mortality between racial groups. (Table 3)

\section{Discussion}

To our knowledge, this large, retrospective cohort study is the first of its kind to directly assess the impact of socioeconomic status, using poverty levels as a primary indicator, on clinical characteristics and outcomes of COVID19 positive patients within the US. Residence in a high poverty area was not associated with an increased risk of COVID-19 related hospitalization, however we found it to 
Table 3 Odds ratio for hospitalization and Hazard Ratio for all-cause mortality among COVID-19 positive patients $(n=3528)$

\begin{tabular}{|c|c|c|c|c|}
\hline & Unadjusted OR (95\% CI) & Adjusted OR* (95\% CI) & Unadjusted HR (95\% CI) & Adjusted $\mathrm{HR}^{\dagger}(95 \% \mathrm{CI})$ \\
\hline Poverty: high vs. low & $0.95(0.83-1.08)$ & $0.98(0.83-1.17)$ & $0.55(0.46-0.66)$ & $0.61(0.51-0.73)$ \\
\hline Age & - & $1.06(1.06-1.07)$ & - & $1.04(1.04-1.05)$ \\
\hline Gender: male vs. female & - & $1.92(1.62-2.27)$ & - & $1.40(1.17-1.67)$ \\
\hline \multicolumn{5}{|l|}{ Race } \\
\hline NHB vs. NHW & - & $1.34(1.07-1.69)$ & - & $1.18(0.94-1.49)$ \\
\hline Other vs. NHW & - & $1.79(1.45-2.20)$ & - & $1.12(0.90-1.39)$ \\
\hline $\mathrm{BMI}>30$ & - & $1.41(1.17-1.70)$ & - & $1.29(1.07-1.56)$ \\
\hline Congestive heart failure & - & $1.53(1.04-2.23)$ & - & - \\
\hline Peripheral vascular disease & - & $1.09(0.68-1.74)$ & - & - \\
\hline Asthma & - & $0.97(0.76-1.25)$ & - & $0.88(0.66-1.17)$ \\
\hline Chronic kidney disease & - & $2.15(1.52-3.03)$ & - & $1.11(0.87-1.40)$ \\
\hline Prior VTE & - & $0.90(0.57-1.41)$ & - & $0.80(0.53-1.22)$ \\
\hline
\end{tabular}

Bold values indicate statistical significance $(\mathrm{p}<0.05)$

$B M I$ body mass index, $C I$ confidence interval, $H R$ hazard ratio, $N H B$ non-Hispanic black, $N H W$ non-Hispanic white, $O R$ Odds ratio, $V T E$ venous thromboembolism

*Adjusted for age (continuous; years), gender, race, BMI > 30, CHF, PVD, asthma, CKD and prior VTE

${ }^{\dagger}$ Adjusted for age (continuous; years), gender, race, BMI > 30, asthma, CKD and prior VTE

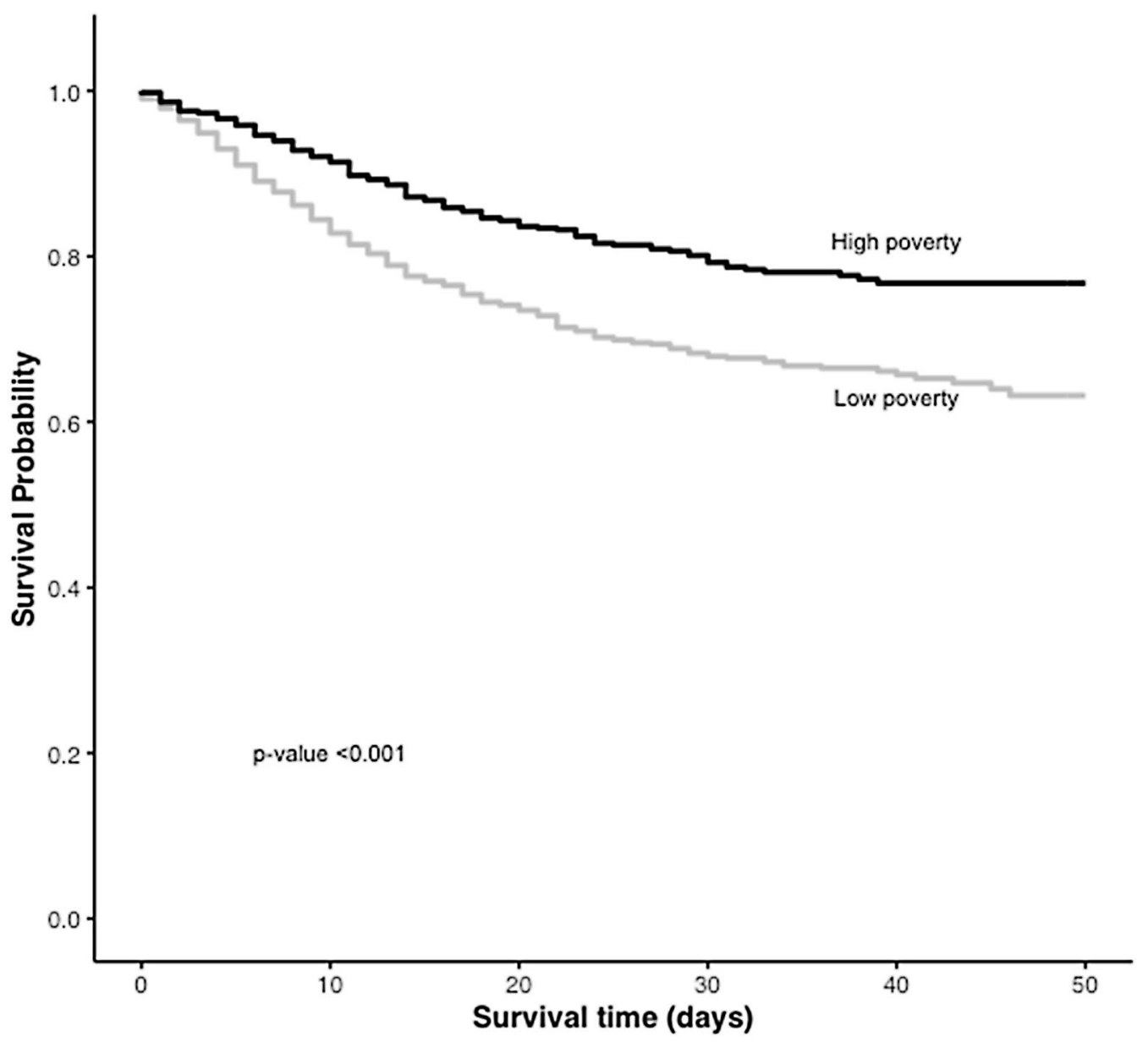

Fig. 1 Survival probability among patients hospitalized with COVID-19 in low poverty and high poverty areas 
be associated with a decreased risk of in-hospital mortality, in particular for male patients.

The decreased risk of mortality among patients living in high poverty areas may be explained by the lacking and conflicting information in the early phase of the pandemic regarding which patients were likely to experience severe COVID-19 disease. Based on recommendations from the $\mathrm{CDC}$ at the time of the study, Emergency Department COVID guidelines recommended increased consideration of admission for patients with cardiovascular disease, diabetes, chronic obstructive pulmonary disease (COPD)/ asthma and CKD due to concern that these comorbidities potentially contributed to COVID-19 disease severity [18, 19]. However, later research has since suggested that these comorbidities may not increase the risk of poor clinical outcomes as much as was initially thought [20]. In particular, this study observed that $\mathrm{CHF}\left(\mathrm{OR}_{\mathrm{adj}}, 1.53 ; 95 \%\right.$ $\mathrm{CI}, 1.04-2.23)$ and $\mathrm{CKD}\left(\mathrm{OR}_{\mathrm{adj}}, 2.15\right.$; $\left.95 \% \mathrm{CI}, 1.52-3.03\right)$ were both associated with increased odds of admission, but were not found to be risk factors for mortality. The prevalence of CHF and CKD was significantly higher among the high poverty cohort compared to those living in low poverty areas, as were other comorbidities such as asthma, diabetes and cardiovascular disease, suggesting this may have influenced the decision to hospitalize these patients while this finally did not bear any impact on risk of mortality.

Furthermore, the decreased risk of mortality among individuals in the high poverty group was shown to be driven by male patients; no significant difference in mortality was observed among female patients between high and low poverty areas. Another reason for hospitalization may have been lack of social support at home to provide close monitoring to detect potential degradation of the clinical status. This may have led to hospitalization of patients from the high poverty group with less severe clinical disease for clinical monitoring. Unfortunately, clinical status at admission, including radiological imaging, oxygenation status and laboratory parameters, were not collected for the purpose of this study. Male gender, a known risk factor for severe COVID-19 disease [20], combined with a higher prevalence of comorbidities in the high poverty group may have led to increased rates of COVID-19 hospitalization of high poverty males who then experienced good clinical outcomes.

Disadvantaged SES is widely associated with adverse health outcomes within the literature [21]. Socioeconomic gradients in health are propagated by social and health disparities which place low income individuals at a higher risk of COVID-19 infection through disparities in living conditions, occupational opportunities, prevalence of comorbidities and financial stability [22]. Our study found evidence to suggest an unequal socioeconomic gradient in the demographic distribution and clinical characteristics of
COVID-19 patients including differences in age, gender and race between poverty groups.

The present study found that among those hospitalized with COVID-19, the mean age of the high poverty group was nearly 5 years younger than the low poverty group. The disparate burden of COVID-19 disease among younger age groups in high poverty areas may be due to a greater percentage of these individuals being employed in occupations or essential jobs that preclude social distancing. These findings may also be explained by the disproportionate percentage of racial minorities in the study population who resided in high poverty areas. Recent evidence has suggested that racial and ethnic minorities are disproportionately affected by COVID-19 at younger ages within the US [5]. Additionally, our data revealed that the percentage of female patients testing positive for COVID-19 was significantly greater in the high poverty cohort ( $49.0 \%$ female) compared to the low poverty cohort ( $42.7 \%$ female). Women are more likely to be employed in essential occupations, such as nurses, teachers or caretakers, that preclude social distancing and increase their risk of COVID-19 exposure [13]. Lower SES women in particular are more likely to be engaged in these professions and may lack the financial stability to leave jobs that place them at a higher risk of exposure, compared to higher SES women [23].

Emerging evidence has raised concerns regarding the disproportionate burden of COVID-19 morbidity and mortality among racial and ethnic minorities within the US, which has been suggested to stem from socioeconomic disadvantages that place low-income individuals at a higher risk of infection [8]. However, our analysis revealed a higher risk of hospitalization for non-Hispanic Black and non-white patients of other race, even after adjusting for socioeconomic status, indicating that the higher risk of hospitalization among minority populations cannot be explained by SES status alone. Minority race was not associated with an increased risk of mortality when adjusting for SES status. These results are consistent with previous studies that observed an increased risk of COVID-19 hospitalization but not mortality between racial groups [5]. The intersectionality of race and socioeconomic factors on COVID-19 transmission and disease severity is still not fully understood and warrants further investigation.

This study has several limitations. It is important to note that income data used in this analysis was not collected at individual-level. It is therefore possible that the individual income of a patient may not match the SES category into which they were placed. However, variance in individual level data has been shown to have minimal impact on aggregate area level socioeconomic indicators [16]. Because income information was only available for ZIP codes contained within the NYC metropolitan area, patients who resided outside of the NYC metropolitan area had to be 
excluded. In addition, the study population was limited to a single NYC healthcare system, which may limit the generalizability of the results to other clinical settings. Lastly, the surge in COVID-19 patient volume at the time of the study may have resulted in decreased time with patients, leading to an increase in missing information on certain covariates such as race, BMI and smoking status.

\section{Conclusions}

This study suggests the existence of an unequal socioeconomic gradient in the demographic and clinical presentation of COVID-19 patients. Further studies are needed to fully assess the influence of SES status on COVID-19 transmission, disease severity and outcomes. Such studies are critical to understand transmission chains, allocate resources and inform public health prevention measures. An emphasis should be placed on collecting additional individual level SES indicators, such as education level, income and residency, in order to further understand the intersectionality of social determinants of health with the COVID-19 pandemic.

Author Contributions Conceptualization and study design: MVG; Data acquisition: MVG, DT, CL, MA, LN and JB; Data analysis: CL, MVG, and MA; Writing —original draft and preparation: CL and MVG; Writing - review, editing and critical input: MVG, MA, JB, DT, ST, LN and LI; Supervision: EG; all authors have contributed significantly and are in agreement with respect to the content of the manuscript. All authors read and approved the final manuscript.

\section{Compliance with Ethical Standards}

Conflict of interest All authors certify that they have no affiliationswith or involvement in any organization or entity with any financial interestor non-financial interest in the subject matter or materials discussed in thismanuscript.

\section{References}

1. Richardson, S., Hirsch, J. S., Narasimhan, M., et al. (2020). Presenting characteristics, comorbidities, and outcomes among 5700 patients hospitalized with COVID-19 in the New York City area. JAMA, 323(20), 2052-2059.

2. Rodriguez-Morales, A. J., Cardona-Ospina, J. A., GutierrezOcampo, E., et al. (2020). Clinical, laboratory and imaging features of COVID-19: A systematic review and meta-analysis. Travel Medicine and Infectious Disease, 34, 101623.

3. Grasselli, G., Zangrillo, A., Zanella, A., et al. (2020). Baseline characteristics and outcomes of 1591 patients infected with SARS-CoV-2 admitted to ICUs of the Lombardy Region, Italy. JAMA, 323(16), 1574-1581.

4. Williamson, E. J., Walker, A. J., Bhaskaran, K., et al. (2020). Factors associated with COVID-19-related death using OpenSAFELY. Nature, 584(7821), 430-436.
5. Price-Haywood, E. G., Burton, J., Fort, D., \& Seoane, L. (2020). Hospitalization and mortality among black patients and white patients with Covid-19. New England Journal of Medicine, 382(26), 2534-2543.

6. Rentsch, C. T., Kidwai-Khan, F., Tate, J. P., et al. (2020). Covid19 by race and ethnicity: A National Cohort Study of 6 Million United States Veterans. medRxiv.

7. Millett, G. A., Jones, A. T., Benkeser, D., et al. (2020). Assessing differential impacts of COVID-19 on Black communities. Annals of Epidemiology, 47, 37-44.

8. Centers for Disease Control and Prevention. Health Equity Considerations and Racial and Ethnic Minority Groups. Retrieved August 11, 2020, from https://www.cdc.gov/coronavirus/2019ncov/community/health-equity/race-ethnicity.html.

9. Hawker, J. I., Olowokure, B., Sufi, F., Weinberg, J., Gill, N., \& Wilson, R. C. (2003). Social deprivation and hospital admission for respiratory infection: An ecological study. Respiratory Medicine, 97(11), 1219-1224.

10. Oestergaard, L. B., Schmiegelow, M. D., Bruun, N. E., et al. (2017). The associations between socioeconomic status and risk of Staphylococcus aureus bacteremia and subsequent endocarditis-A Danish nationwide cohort study. BMC Infectious Diseases, 17(1), 589.

11. Rutter, P. D., Mytton, O. T., Mak, M., \& Donaldson, L. J. (2012). Socio-economic disparities in mortality due to pandemic influenza in England. International Journal of Public Health, 57(4), 745-750.

12. Das, A., Ghosh, S., Das, K., Basu, T., Das, M., \& Dutta, I. (2020). Modeling the effect of area deprivation on COVID-19 incidences: A study of Chennai megacity, India. Public Health, $185,266-269$.

13. Khalatbari-Soltani, S., Cumming, R. C., Delpierre, C., \& KellyIrving, M. (2020). Importance of collecting data on socioeconomic determinants from the early stage of the COVID-19 outbreak onwards. Journal of Epidemiology and Community Health, 74(8), 620-623.

14. Toprani, A., \& Hadler, J. (2013). Selecting and applying a standard area-based socioeconomic status measure for public health data: Analysis for New York City. New York City: Department of health and mental hygiene: Epi Research Report.

15. Krieger, N., Chen, J. T., Waterman, P. D., Soobader, M. J., Subramanian, S. V., \& Carson, R. (2003). Choosing area based socioeconomic measures to monitor social inequalities in low birth weight and childhood lead poisoning: The Public Health Disparities Geocoding Project (US). Journal of Epidemiology and Community Health, 57(3), 186-199.

16. Berkowitz, S. A., Traore, C. Y., Singer, D. E., \& Atlas, S. J. (2015). Evaluating area-based socioeconomic status indicators for monitoring disparities within health care systems: Results from a primary care network. Health Services Research, 50(2), 398-417.

17. Geronimus, A. T., \& Bound, J. (1998). Use of census-based aggregate variables to proxy for socioeconomic group: Evidence from national samples. American Journal of Epidemiology, 148(5), 475-486.

18. New York City Department of Health and Mental Hygiene. Health Alert \# 6: COVID-19 Updates for New York City. Retrieved August 29, 2020, from https://www1.nyc.gov/assets/doh/downl oads/pdf/han/alert/2020/covid-19-03152020.pdf.

19. Centers for Disease Control and Prevention. People with Certain Medical Conditions. Retrieved July 23, 2020, from https ://www.cdc.gov/coronavirus/2019-ncov/need-extra-preca utions/people-with-medical-conditions.html?CDC_AA_refVa l=https $\% 3 \mathrm{~A} \% 2 \mathrm{~F} \% 2 \mathrm{Fwww} . c d c . g o v \% 2 \mathrm{Fcoronavirus} \% 2 \mathrm{~F} 201$ 9-ncov\%2Fneed-extra-precautions\%2Fgroups-at-higher-risk.html.

20. van Gerwen, M., Alsen, M., Little, C., et al. (2020). Risk factors and outcomes of COVID-19 in New York City; a 
retrospective cohort study. Journal of Medical Virology. https:// doi.org/10.1002/jmv.26337.

21. Lantz, P. M., Lynch, J. W., House, J. S., et al. (2001). Socioeconomic disparities in health change in a longitudinal study of US adults: The Role of health-risk behaviors. Social Science \& Medicine, 53(1), 29-40.

22. Evans, G. W., \& Kim, P. (2010). Multiple risk exposure as a potential explanatory mechanism for the socioeconomic status-health gradient. Annals of the New York Academy of Sciences, 1186, 174-189.
23. England, P. (2010). The gender revolution: Uneven and stalled. Gender \& Society, 24(2), 149-166.

Publisher's Note Springer Nature remains neutral with regard to jurisdictional claims in published maps and institutional affiliations. 